\title{
MEASURING THE DECELERATION PARAMETER FROM COSMOLOGICAL REDSHIFT DISTORTION OF GALAXY CORRELATION FUNCTION AT SMALL REDSHIFT
}

\author{
TAKAHIRO T. NAKAMURA \\ Department of Physics, University of Tokyo, Tokyo 113, Japan
}

We present a formula to infer the value of the deceleration parameter $q_{0}$ from the anisotropy in the galaxy correlation function at small redshift:

$$
1+q_{0} \simeq \beta_{0}^{2} \frac{d_{1}}{\Delta}+\left(\beta_{0}+\frac{1}{3} \beta_{0}^{2}\right) \frac{d_{2}}{\Delta}+\left(1+\frac{6}{7} \beta_{0}+\frac{3}{35} \beta_{0}^{2}\right) \frac{d_{3}}{\Delta},
$$

where $\beta_{0}=\Omega_{0}^{0.6} / b$, and $d_{1}, d_{2}, d_{3}$ and $\Delta$ are written in terms of the multipole components of the redshift-space correlation function $\xi_{\mathrm{s}}(s, \nu)$ :

$$
\zeta_{2 l}(s ; z):=\left(2 l+\frac{1}{2}\right) \int_{-1}^{1} d \nu P_{2 l}(\nu) \xi_{\mathrm{s}}(s, \nu ; z)
$$

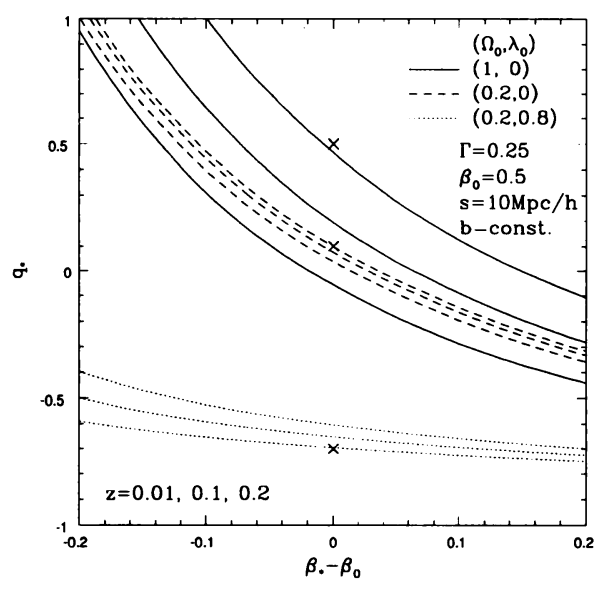

Figure 1. Inferred value $q_{*}$ of the deceleration parameter $q_{0}$ from equation (1), versus $\beta_{*}$ which is substituted for $\beta_{0}=\Omega_{0}^{0.6} / b$ in the right hand sides of equation (1). The crosses indicate the location of the true values of $q_{0}=\frac{1}{2} \Omega_{0}-\lambda_{0}$.

The present method does not require any assumption of the shape and amplitude of the underlying fluctuation spectrum, and thus can be applied to future redshift surveys of galaxies including the Sloan Digital Sky Survey. More details are found in Nakamura, Matsubara \& Suto (1998, ApJ 493 in press) 